\title{
Easter Island volume
}

\section{Fedor Bogomolov ${ }^{1,2} \cdot$ Ivan Cheltsov ${ }^{2,3} \cdot$ Adrien Dubouloz $^{4} \cdot$ Alvaro Liendo $^{5}$}

Published online: 9 August 2019

(C) Springer Nature Switzerland AG 2019

On the 17th of December 2016, twenty four mathematicians arrived to Easter Island. Alvaro Liendo and Sukhendu Mehrotra came from Chile, Sione Ma'u came from Auckland in New Zealand, and four mathematicians came from Japan: Will Donovan, Kento Fujita, Yoshinori Gongyo, and Yujiro Kawamata. Five mathematicians flew from the States: Valery Alexeev came from Athens in Georgia, Fedor Bogomolov came from New York, Joe Cutrone came from Baltimore, Alexander Duncan came from Columbia in South Carolina, and Joaquin Moraga came from Salt Lake City. Nikita Kalinin and Ernesto Lupercio both came from Mexico City, DongSeon Hwang and Dima Sakovics came from South Korea, and the remaining eight mathematicians traveled from Europe: Elena Bunkova (Moscow), Ivan Cheltsov (Edinburgh), Lucas das Dores (Liverpool), Adrien Dubouloz (Dijon), Ilia Itenberg (Paris), Angelo Lopez (Rome), Grigory Mikhalkin (Geneva), and Navid Nabijou (London). All of them came to the island to participate in a conference on Algebraic Geometry. This volume carries 26 papers related to this conference.

Why Easter Island? We wanted to show that people can do Mathematics everywhere including the most remote places. Easter Island fits this goal perfectly, since it

$凶 \quad$ Ivan Cheltsov

I.Cheltsov@ed.ac.uk

Fedor Bogomolov

bogomolo@cims.nyu.edu

Adrien Dubouloz

Adrien.Dubouloz@u-bourgogne.fr

Alvaro Liendo

aliendo@inst-mat.utalca.cl

1 Courant Institute of Mathematical Sciences, New York University, 251 Mercer Street, New York, NY 10012, USA

2 Laboratory of Algebraic Geometry, National Research University Higher School of Economics, 6 Usacheva Street, Moscow, Russia 117312

3 School of Mathematics, The University of Edinburgh, Edinburgh EH9 3FD, UK

4 Institut de Mathématiques de Bourgogne - UMR 5584 du CNRS, Université de Bourgogne, 9 Avenue Alain Savary, 21078 Dijon, France

5 Instituto de Matemática y Física, Universidad de Talca, Casilla 721, Talca, Chile 
is one of the world's most isolated inhabited islands: the nearest continental point is 3512 kilometers away in Chile; the closest inhabited islands are three Juan Fernández Islands (1850 kilometers to the east) and Pitcairn Island (2075 kilometers to the west). The only island that competes with Easter Island for the title of being the most remote is Tristan da Cunha in the southern Atlantic, which does not have an airport and can only be reached by sea.

Geometrically, Easter Island is a triangle whose sides are 16 kilometers, 18 kilometers, and 24 kilometers. Its area is approximately 164 square kilometers, and its longest side is the south-east coast of the island. Close to the vertices of this triangle, there are three extinct volcanoes: Rano Kau, Rano Raraku, and Rano Aroi. The highest point of Easter Island is the summit of Terevaka, which is 507 meters above the sea level. The island is very small and flat: it is possible to walk around the island in one day.

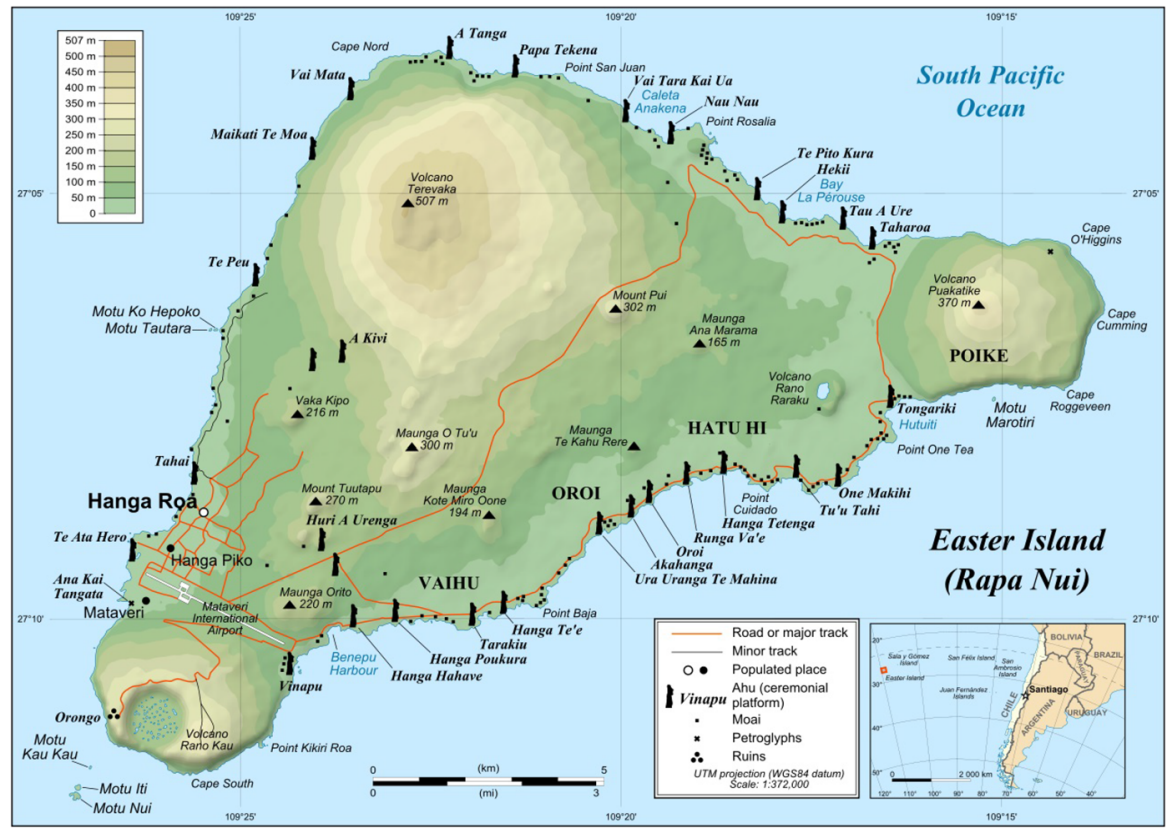

From Wikipedia, courtesy of Eric Gaba

The history of Easter Island is interesting, and can be even called rich for such a lonely remote small place. It was first settled by a small group of Polynesians about or shortly after $400 \mathrm{CE}$. At this time, the island was covered by great palm forest. In the years following the initial settlement, islanders were cutting down trees, making canoes, and catching fish. A small initial population of Easter Island grew to a developed society, whose members built thousands of huge statues, called Moai, which was an enormous achievement for island's inhabitants. This mega project played a gloomy role in island's history: the islanders crucially overused their resources, so that the large trees completely disappeared from the island. As a result, the islanders were unable to build boats, and the loss of forests also led to soil erosion, causing lack of fresh water, which severely damages agriculture. During long stagnation period, islanders ate all birds and dogs, and catched all fish that lived close to the island, so 
that the only available food left were chicken, bananas, sweet potatoes, sugar cane and rats. Then, on Easter Sunday in 1722, the island was discovered by the Dutch explorer Jacob Roggeveen, who gave the island its name.

By the time of Roggeveen arrival, there was not a single tree on Easter Island, and its population was around 2000 people, who lived in a very poor (but seemingly stable) condition, and all ancient knowledge was completely forgotten. The next contact with Europeans was a short visit of two Spanish ships in 1770. After this the British explorer James Cook visited Easter Island in 1774. The contacts with Europeans ignited political and social crisis in local community: the whole Easter Island civilization collapsed in a short time: aristocracy was destroyed, all Moais had been knocked over as a part of internal conflicts between islanders, who split into tribes and started to fight (and occasionally eat) each other. Nevertheless, the attitude to foreigners remained positive for quite some time: when the French explorer Jean-François de La Pérouse visited the island in 1786, he was met with a warm welcome. This, of course, could not last forever: when Russian admiral Otto von Kotzebue visited Easter Island in 1816, the locals started to throw stones at him and his crew. Puzzled by this hostile behavior, von Kotzebue wrote in his memoirs:

"The mistrustful attitude of islanders made me think that they probably had a quarrel with Europeans at some point, and Europeans destroyed their statues in revenge. I was also surprised that I did not see any women in the water or on the coast, while previous visitors complained about their bothering behavior. This convinced me that of course Europeans recently did here horrible things."

von Kotzebue was right: in 1805, a group of Americans arrived to Easter Island and took 12 hostages after a bloody fight. Later a series of similar devastating events killed or removed most of the population of Easter Island. The worst of them happened in 1862, when Peruvian slave raiders violently abducted around 1500 men and women. Later 15 survivors returned to Easter Island from Peru only to bring smallpox disease with them, which created a devastating epidemic.

In 1868, French mariner Jean-Baptiste Dutrou-Bornier, the agent of Tahiti based company the Maison Brander, began buying up land from islanders with legal help from Catholic mission. Eventually, he bought up all of the island apart from a small piece of land in around Hanga Roa, and turned Easter Island in one big sheep ranch. Islanders, completely deprived of their land, had to live in Hanga Roa as prisoners. Since the ranch did not need many people to take care of the sheep, Dutrou-Bornier moved hundreds of islanders to Mangareva and Tahiti: only 111 of them remained on the island in 1877.

On the 9th of September 1888, Chile annexed Easter Island. But this did not help its native people: Chile leased the island to the Williamson-Balfour Company, which continued to run it as a sheep ranch until 1953 (the company constructed a wall around Hanga Roa to keep local people in the village). Between 1953 and 1966, the island was managed by the Chilean Navy. In 1966, all islanders were granted Chilean citizenship and became able to move freely in the island. In 2007, Easter Island gained special constitutional status that brought islanders peace and prosperity. Now, with a single exception of posh Hangaroa Eco Village Spa which hosted our conference, all land and local businesses on the island belong to the natives. Since tourism thrives, all 
islanders have very stable income that allows them to have a middle class life style, which was simply unimaginable few generations ago.

Relaxed life style and spiritual happiness of the islanders created very friendly atmosphere during our meeting, which helped to organize this complete volume: every participant submitted a paper. Since the collapse of Easter Island's economics serves as a standard model in many problems on population dynamics, we also included three papers that describe the ecosystem of Easter Island using differential equations. In total, fifty one mathematicians contributed 26 papers to this volume. Let us describe their contribution.

Let $S$ be a projective surface defined over $\mathbb{C}$, and let $B$ be an $\mathbb{R}$-divisor whose coefficients lie in a fixed set $\Sigma$ that satisfies the descending chain condition. Suppose that the pair $(S, B)$ has at most log canonical singularities, and that the log canonical divisor $K_{S}+B$ is ample. Then the set

$$
\left\{\left(K_{S}+B\right)^{2}:(S, B) \text { is log canonical, and } K_{S}+B \text { is ample }\right\}
$$

also satisfies the descending chain condition, so that it attains the absolute minimum, a positive real number. Long time ago, Valery Alexeev and Shigefumi Mori found an effective lower bound for this number, which is unrealistically small. Recently, Alexeev and Wenfei Liu found surfaces with the smallest known volumes in the cases when $\Sigma=\{0\}$ and $\Sigma=\{0,1\}$. Their examples were obtained by blow-ups and contractions of the four-line configuration in the plane. In the paper Log surfaces of Picard rank one from four lines in the plane, Alexeev and Liu found the smallest positive volume and the smallest accumulation point of volumes of log canonical surfaces with Picard number one obtained in this way.

Let $X$ be a scheme of finite type over an arbitrary field. To measure the minimal homological complexity of the category $\mathrm{D}^{b}(\operatorname{coh}(X))$, Raphaël Rouquier introduced a new invariant and proved that it is always at least the Krull dimension of the scheme $X$ (with equality in certain cases). This invariant is now called the Rouquier dimension of the scheme $X$. Recently Dima Orlov posed the following

Conjecture Suppose that $X$ is smooth and projective. Then the Rouquier dimension of the scheme $X$ is equal to the Krull dimension of the scheme $X$.

Orlov also proved this conjecture in dimension 1 (for smooth projective curves). In higher dimensions, Orlov's conjecture seems to be very difficult to address, and all supporting evidence for it comes from individual constructions specialized to particular examples. In the paper The toric Frobenius morphism and a conjecture of Orlov, Matthew Ballard, Alexander Duncan, and Patrick McFaddin expand the set of varieties satisfying Orlov's conjecture. To do this, they combine the Bondal-Uehara method for producing exceptional collections on toric varieties with an earlier result of Ballard and Favero. Moreover, Ballard, Duncan, and McFaddin prove Orlov's conjecture for a (smooth and projective) scheme $X$ in the case when the Bondal-Uehara method produces a tilting bundle on it.

As we already mentioned above, the deforestation of Easter Island led to a collapse of its ecosystem and population numbers. In the paper Ecological collapse of Easter 
Island and the role of price fixing, William and Wesley Basener review some of the factors that have been studied which may have contributed to the collapse. They show that the function for the harvesting rate is an important factor in dynamics that can lead to total collapse of the resource. In particular, they prove that harvesting rate functions resulting in total collapse correspond to fixed prices of the resources.

In the paper Homomorphisms of multiplicative groups offields preserving algebraic dependence, Fedor Bogomolov, Marat Rovinsky, and Yuri Tschinkel study homomorphisms of multiplicative groups of fields preserving algebraic dependence and show that such homomorphisms give rise to valuations.

A real algebraic variety $(X, c)$ is a complex algebraic variety $X$ equipped with an anti-holomorphic involution $c: X \rightarrow X$, which is called a real structure. The fixed point set of $c$ is the real part of $X$, which is usually denoted by $\mathbb{R} X$. A real algebraic variety is called finite if its real part consists of finitely many points. Let $C$ be a finite real algebraic curve in the complex projective plane. Then its degree must be even, so that we have $\operatorname{deg} C=2 k$ for some positive integer $k$. Let

$$
\delta(k)=\max \left\{|\mathbb{R} C|: C \text { a finite real algebraic curve in } \mathbb{P}^{2} \text { such that } \operatorname{deg} C=2 k\right\}
$$

and

$$
\delta_{g}(k)=\max \left\{|\mathbb{R} C|: \begin{array}{l}
C \text { a finite real algebraic curve in } \mathbb{P}^{2} \\
\text { of genus } g \text { such that } \operatorname{deg} C=2 k
\end{array}\right\},
$$

where $g$ is a non-negative integer. In the paper Real algebraic curves with large finite number of real points, Erwan Brugallé, Alex Degtyarev, Ilia Itenberg, and Frédéric Mangolte improve the known upper and lower bounds for $\delta(k)$ and $\delta_{g}(k)$, and construct close to optimal curves of small degree.

In the paper On the problem of differentiation of hyperelliptic functions, Elena Bunkova describes a construction that leads to an explicit solution of the problem of differentiation of hyperelliptic functions. Namely, she gives explicit generators for the Lie algebra Der $\mathcal{F}$ of derivations of the fields of hyperelliptic functions $\mathcal{F}$ of genus $g$ for $g \in\{1,2,3\}$.

In the paper Fujita decomposition over higher dimensional base, Fabrizio Catanese and Yujiro Kawamata generalize a result of Takao Fujita, on the decomposition of Hodge bundles over curves, to the case of a higher dimensional base.

Let $X$ be a smooth Fano variety. For a sufficiently large and sufficiently divisible integer $k$, consider a basis $s_{1}, \ldots, s_{d_{k}}$ of the vector space $H^{0}\left(\mathcal{O}_{X}\left(-k K_{X}\right)\right)$, where $d_{k}=h^{0}\left(\mathcal{O}_{X}\left(-k K_{X}\right)\right)$. For this basis, consider the $\mathbb{Q}$-divisor

$$
\frac{1}{k d_{k}} \sum_{i=1}^{d_{k}}\left\{s_{i}=0\right\} \sim_{\mathbb{Q}}-K_{X}
$$

Any $\mathbb{Q}$-divisor obtained in this way is called a $k$-basis type (anticanonical) divisor. Let

$$
\delta_{k}(X)=\sup \left\{\lambda \in \mathbb{Q}: \begin{array}{l}
\text { the } \log \text { pair }(X, \lambda D) \text { is } \log \text { canonical } \\
\text { for every } k \text {-basis type } \mathbb{Q} \text {-divisor } D \sim_{\mathbb{Q}}-K_{X}
\end{array}\right\} .
$$


Then let

$$
\delta(X)=\limsup _{k \in \mathbb{N}} \delta_{k}(X) .
$$

If $\delta(X)>1$, then $X$ admits a Kähler-Einstein metric. In the paper Delta invariants of smooth cubic surfaces, Ivan Cheltsov and Kewei Zhang prove that $\delta(X) \geqslant 6 / 5$ in the case when $X$ is a smooth cubic surface in $\mathbb{P}^{3}$. This shows that all smooth cubic surfaces in $\mathbb{P}^{3}$ are Kähler-Einstein, which is a classical result by Gang Tian.

Let $X$ be a smooth threefold such that $-K_{X}$ is numerically effective (nef) and $-K_{X}^{3}>0$ (big). Then $X$ is said to be a weak Fano threefold. Weak Fano threefolds with Picard rank 2 arise naturally in the study of birational maps between smooth Fano threefolds with Picard rank 1, which leads to the (old) problem of their classification. This classification, started by Jahnke, Peternell, and Radloff long time ago, is based on the classical approach to birational geometry of Fano threefolds originated in the works of Vasily Iskovskikh and Kiyohiko Takeuchi. Its first (and probably most important) step is to create a list of numerical possibilities for extremal contraction from $X$. Relatively recently, Joseph Cutrone and Nicholas Marshburn completed this numerical classification and provided huge tables that capture geometric realizations of already existing weak Fano threefolds. Cutrone and Marshburn also gave geometric constructions for many new numerical examples. Later, more geometric constructions were given by them and Maxim Arap, Jeremy Blanc, Ivan Cheltsov, Stephane Lamy, and Constantin Shramov, which filled almost all existence cells in Cutrone-Marshburn tables. However, the actual geometric existence of some cases is an open problem. In the paper A weak Fano threefold arising as a blowup of a curve of genus 5 and degree 8 on $\mathbb{P}^{3}$, Joseph Cutrone, Michael Limarzi, and Nicholas Marshburn sort out one such case: they construct a smooth weak Fano threefold of Picard number two with small anti-canonical morphism that arises as a blowup of a smooth curve of genus 5 and degree 8 in $\mathbb{P}^{3}$.

In the paper Perverse schobers on Riemann surfaces: constructions and examples, Will Donovan studies perverse sheaves of categories on Riemann surfaces. Among other results, he constructs, for certain wall crossings in geometric invariant theory, a schober on the complex plane, singular at each imaginary integer. He also suggests an application to mirror symmetry.

Let $S$ be a smooth projective surface defined over the field of complex numbers, let $G$ be a finite group, and let $\rho: G \rightarrow \operatorname{Aut}(S)$ be a monomorphism. Then the pair $(S, \rho)$ is said to be a $G$-surface. Given two $G$-surfaces $(S, \rho)$ and $\left(S^{\prime}, \rho^{\prime}\right)$, we say that a rational map $\varphi: S \rightarrow S^{\prime}$ is $G$-rational if for any $g \in G$ the following diagram commutes for some $g^{\prime} \in G$ :

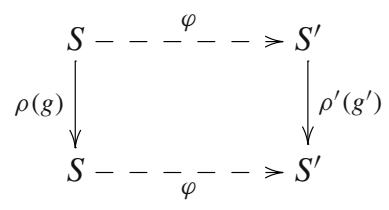

A $G$-surface $(S, \rho)$ is said to be minimal if every birational $G$-morphism $S \rightarrow S^{\prime}$ is an isomorphism. For instance, if $S$ is a smooth Del Pezzo surface such that $\operatorname{Pic}^{G}(S) \cong \mathbb{Z}$, then the $G$-surface $(S, \rho)$ is clearly minimal. 
Definition Let $(S, \rho)$ be a minimal $G$-surface such that $S$ is a smooth Del Pezzo surface, and $\operatorname{Pic}^{G}(S) \cong \mathbb{Z}$. Then $(S, \rho)$ is said to be $G$-birationally rigid ( $G$-birationally super-rigid, respectively) if there is no $G$-birational map from $S$ to any other minimal $G$-surface (and the group of $G$-biregular automorphisms of the surface $S$ coincides with the group of its $G$-birational automorphisms, respectively).

If $(S, \rho)$ is a $G$-surface such that $S$ is a smooth del Pezzo surface, $K_{S}^{2} \leqslant 3$ and $\operatorname{Pic}^{G}(S) \simeq \mathbb{Z}$, then $(S, \rho)$ is $G$-birationally rigid by the classical theorem of Beniamino Segre and Yuri Manin. In the paper G-birational superrigidity of Del Pezzo surfaces of degree 2 and 3, Lucas das Dores and Mirko Mauri refine this result by determining which $G$-surfaces in the Segre-Manin theorem are $G$-birationally superrigid and which are not.

In the paper Exotic $\mathbb{G}_{a}$-quotients of $\mathrm{SL}_{2} \times \mathbb{A}_{1}$, Adrien Dubouloz proves that every affine threefold that is given in $\mathbb{C}^{4}$ by

$$
x^{n} z=y^{m}-t^{r}+x h(x, y, t)
$$

is the algebraic quotient of proper Zariski locally trivial $\mathbb{G}_{a}$-action on $\operatorname{SL}(2, \mathbb{C}) \times \mathbb{C}^{1}$. Here, $n, m$, and $r$ are co-prime natural numbers such that $n \geqslant 2$, and $h(x, y, t)$ is a polynomial in $\mathbb{C}[x, y, t]$ such that $h(0,0,0) \neq 0$.

In the paper The effect of tree diffusion in a two-dimensional continuous model for Easter Island, István Faragó, Róbert Horváth, and Bálint Takács consider a twodimensional continuous model that describes the ecology of Easter Island. They show that the increase of the parameter corresponding to the diffusion of trees on the island has a stabilizing effect on the system, potentially preventing the collapse of island's ecology.

Let $X$ be a normal, complex, projective algebraic variety of dimension $d$, and let $\Delta$ be an effective $\mathbb{Q}$-divisor on $X$ such that $-\left(K_{X}+\Delta\right)$ is an ample $\mathbb{Q}$-Cartier divisor, and the $\log$ pair $(X, \Delta)$ has at most log canonical singularities. Let

$$
\iota_{(X, \Delta)}=\min \left\{-\left(K_{X}+\Delta\right) \cdot C: C \text { is a rational curve in } X\right\} .
$$

If $X$ is smooth and $\Delta=0$, then $\iota_{(X, \Delta)} \leqslant d+1$ by a classical result of Shigefumi Mori. Relatively recently, Osamu Fujino proved that $\iota_{(X, \Delta)} \leqslant 2 d$. Then Bonavero, Casagrande, Debarre, and Druel posed the following

Conjecture Let $\rho_{X}$ be the rank of the Picard group of $X$. Then

$$
\iota_{(X, \Delta)} \leqslant \frac{d}{\rho_{X}}+1 .
$$

Moreover, the equality holds if and only if $X \cong\left(\mathbb{P}^{1}\right)^{d}$ and $\Delta=0$.

In the paper The generalized Mukai conjecture for toric log Fano pairs, Kento Fujita proves this conjecture in the case when $X$ is toric, and $\Delta$ is a torus invariant $\mathbb{Q}$-divisor.

Let us remind the reader that Kollár's injectivity theorem for semi-ample line bundles is the following classical result: 
Theorem Let $X$ be an $n$-dimensional smooth projective variety over an algebraically closed field of characteristic zero, let $\mathcal{L}$ be a semi-ample line bundle on $X$, and let $S$ be a non-zero section in $H^{0}\left(X, \mathcal{L}^{\otimes \ell}\right)$ for some positive integer $\ell$. Then the map

$$
\times s: H^{i}\left(X, \omega_{X} \otimes \mathcal{L}^{\otimes m}\right) \rightarrow H^{i}\left(X, \omega_{X} \otimes \mathcal{L}^{\otimes(\ell+m)}\right)
$$

induced by the tensor product with $s$ is injective for all $m \geqslant 1$ and $i \geqslant 0$, where $\omega_{X}$ is the canonical line bundle on $X$.

In the paper Kollár's type injectivity theorem for globally F-regular varieties, Yoshinori Gongyo and Shunsuke Takagi prove the following analogue of this theorem for varieties defined over a field of positive characteristic.

Theorem Let $X$ be an $n$-dimensional globally $F$-regular projective variety over an $F$-finite field of characteristic $p>0$, let $\mathcal{L}$ be a semi-ample line bundle on $X$, and let $s$ be a non-zero section in $H^{0}\left(X, \mathcal{L}^{\otimes \ell}\right)$ for some positive integer $\ell$. Then the map

$$
\times s: H^{i}\left(X, \omega_{X} \otimes \mathcal{L}^{\otimes m}\right) \rightarrow H^{i}\left(X, \omega_{X} \otimes \mathcal{L}^{\otimes(\ell+m)}\right)
$$

induced by the tensor product with $s$ is injective for all $m \geqslant 1$ and $i \geqslant 0$, where $\omega_{X}$ is the canonical line bundle on $X$.

In the paper Nearly Frobenius algebras, Ana González, Ernesto Lupercio, Carlos Segovia, and Bernardo Uribe study nearly Frobenius algebras, generalizations of Frobenius algebras which appear naturally in topology. They present foundational results about nearly Frobenius algebras and consider some applications in geometry, topology, and representation theory.

In the paper A curve-detecting formula for projective surfaces, DongSeon Hwang presents an intersection-theoretic formula about curves on projective surfaces in terms of lattices with special emphasis on minimal resolutions of $\mathbb{Q}$-homology projective planes. This formula can be used to detect the existence or nonexistence of curves with given intersection properties.

In the paper Tropical formulae for summation over a part of $\mathrm{SL}(2, \mathbb{Z})$, Nikita Kalinin and Mikhail Shkolnikov consider the sum of the powers of the triangle inequality defects for the lattice parallelograms (in the first quadrant) of area one. To be precise, let

$$
f(a, b, c, d)=\sqrt{a^{2}+b^{2}}+\sqrt{c^{2}+d^{2}}-\sqrt{(a+c)^{2}+(b+d)^{2}}
$$

and

$$
F(s)=\sum f(a, b, c, d)^{s},
$$

where the sum is taken over all non-negative integers $a, b, c$, and $d$ such that $a d-b c=1$. Kalinin and Shkolnikov prove that $F(s)$ converges when $s>1$ and diverges at $s=1 / 2$. Using tropical method they also establish the following formula:

$$
\sum \frac{1}{(a+b)^{2}(c+d)^{2}(a+b+c+d)^{2}}=\frac{1}{3},
$$


where the range of summation is as above.

In the paper Ordinary differential equations and Easter Island, Lorelei Koss gives a survey of ordinary differential equation models investigating environmental and sustainability issues in the history of Easter Island.

A $\mathbb{T}$-variety is an algebraic variety endowed with an effective action of an algebraic torus. In the paper The fundamental group of log terminal $\mathbb{T}$-variety, Antonio Laface, Alvaro Liendo, and Joaquin Moraga study the fundamental group of varieties with log terminal singularities endowed with an algebraic torus $\mathbb{T}$-action. In particular, they prove the simple connectedness of the spectrum of the Cox ring of a complex Fano variety, and compute the fundamental group of rational $\log$ terminal $\mathbb{T}$-varieties of complexity one.

Let $X$ be a smooth projective variety defined over the field of complex numbers, and let $H$ be an ample divisor on $X$. An Ulrich vector bundle for the polarized pair $(X, H)$ is a vector bundle $\mathcal{E}$ on the variety $X$ such that

$$
H^{i}(\mathcal{E}(-p H))=0
$$

for every $i \geqslant 0$ and every positive integer $p \leqslant \operatorname{dim} X$. Even though Ulrich bundles have received a lot of attention in recent years, the basic existence question on any $X$ is still open even in dimension two. Several classes of smooth surfaces do carry an Ulrich vector bundle: $K 3$ surfaces, abelian surfaces, bielliptic surfaces, Enriques surfaces, del Pezzo surfaces, hypersurfaces and complete intersections, and some ruled surfaces. In the paper On the existence of Ulrich vector bundles on some surfaces of maximal Albanese dimension, Angelo Lopez proves the existence of a simple rank two Ulrich vector bundle $\mathcal{E}$ for the pair $(X, H)$ in the case when the following three conditions are satisfied:

- $X$ is a surface with maximal Albanese dimension;

- $\chi\left(\mathcal{O}_{S}\right)=0$;

- $H$ is a very ample divisor on $X$ such that $h^{1}\left(\mathcal{O}_{S}(H)\right)=0$.

Let $S$ be a smooth $K 3$ surface, and let $M$ be a smooth and projective $2 n$-dimensional moduli space of stable coherent sheaves on the surface $S$. Then there exists a rank $2 n-2$ reflexive hyperholomorphic sheaf $E_{M}$ on the product $M \times M$, whose fiber over a non-diagonal point $(F, G)$ is $\operatorname{Ext}^{1}(F, G)$. The sheaf $E_{M}$ can be deformed along some twistor path to a sheaf $E_{X}$, over the Cartesian square of every Kähler manifold $X$ deformation equivalent to $M$. In the paper Rigid hyperholomorphic sheaves remain rigid along twistor deformations of the underlying hyperkähler manifold, Eyal Markman, Sukhendu Mehrotra, and Misha Verbitsky prove that the sheaf $E_{X}$ is infinitesimally rigid, and the isomorphism class of the Azumaya algebra $\mathcal{E} n d\left(E_{X}\right)$ is independent of the twistor path chosen.

In the paper Relating transfinite diameters using an Okounkov body, Sione Ma'u studies some notions of pluripotential theory using an Okounkov body.

In the paper Examples of tropical-to-Lagrangian correspondence, Grisha Mikhalkin associates Lagrangian submanifolds in symplectic toric varieties to certain tropical curves inside the convex polyhedral domains of $\mathbb{R}^{n}$ that appear as the images of the 
moment map of the toric varieties. In particular, for the case $n=2$, Grisha reproves Givental's theorem on Lagrangian embeddability of non-oriented surfaces to $\mathbb{C}^{2}$.

Let $X$ be a smooth projective variety. Givental's Lagrangian cone $\mathcal{L}_{X}$ is a Lagrangian submanifold of a symplectic vector space which encodes the genus-zero GromovWitten invariants of the variety $X$. Building on work of Sasha Braverman, Tom Coates obtained the Lagrangian cone as the push-forward of a certain class on the moduli space of stable maps to $X \times \mathbb{P}^{1}$. In the paper The fundamental solution matrix and relative stable maps, Navid Nabijou recasts this construction in its natural context, namely the moduli space of stable maps to $X \times \mathbb{P}^{1}$ relative the divisor $X \times \infty$. He finds that the resulting push-forward is another familiar object, namely the transform of the Lagrangian cone under the action of the fundamental solution matrix. Nabijou also uses a hidden polynomiality property implied by his construction to obtain a sequence of universal relations for the Gromov-Witten invariants.

In 1917, Hans Frederick Blichfeldt classified all finite subgroups in $\mathrm{PGL}_{3}(\mathbb{C})$ and $\mathrm{PGL}_{4}(\mathbb{C})$. In the paper $G$-birational rigidity of the projective plane, Dima Sakovics describes all finite subgroups $G \subset \mathrm{PGL}_{3}(\mathbb{C})$ such that $\mathbb{P}^{2}$ is $G$-birationally rigid. His classification implies that $\mathbb{P}^{2}$ is $G$-birationally rigid if and only if the following two conditions are satisfied:

- the plane $\mathbb{P}^{2}$ does not contain $G$-fixed points;

- the group $G$ is isomorphic neither to $\mathfrak{A}_{4}$ nor to $\mathfrak{S}_{4}$.

Although Easter Island does not have any research mathematical centers, we feel that the location is an ideal place for an international scientific meeting. This is confirmed by the Easter Island Volume, which illustrates how fruitful our conference was. We hope that the reader will enjoy reading its contributions.

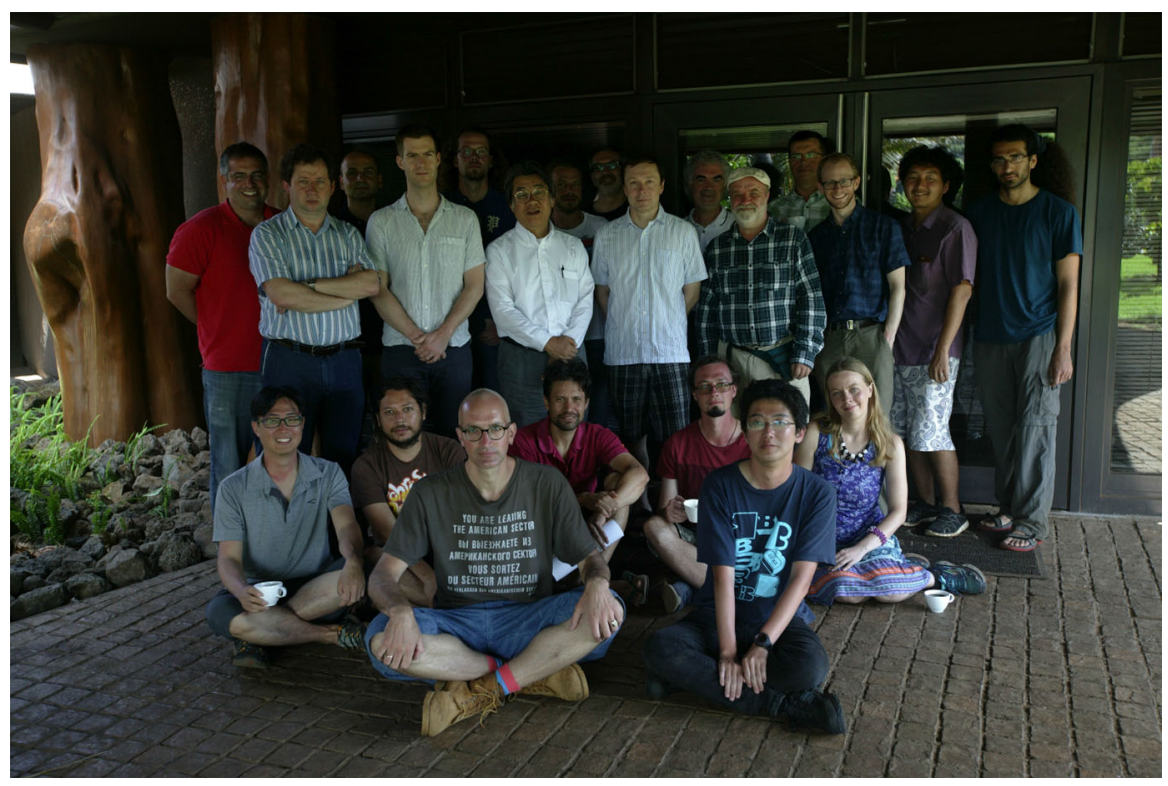


Acknowledgements On behalf of the participants of this conference on Algebraic Geometry, we thank Universidad de Talca for its kind support.

Publisher's Note Springer Nature remains neutral with regard to jurisdictional claims in published maps and institutional affiliations. 